\title{
Propriedades Psicométricas do Test de Empatía Cognitiva y Afectiva (TECA) em Estudantes Universitários Brasileiros
}

\author{
Psychometric Properties of the Cognitive and Affective Empathy Test (CAET) in \\ Brazilian University Students
}

\author{
Julio Cesar de Freitas ${ }^{1}$ e Guilhermina Lobato Miranda ${ }^{2}$
}

\begin{abstract}
Resumo
Neste artigo apresentamos os resultados do processo de validação do "Test de Empatía Cognitiva y Afectiva (TECA)" para uma amostra de 256 estudantes universitários brasileiros. Apresentamos o processo de tradução e retroversão da escala original, a sensibilidade dos itens, a validade fatorial da escala e a fiabilidade das suas quatro dimensões e comparámos os nossos resultados com os do estudo original. Na globalidade os itens que compõem a escala mostraram ser sensíveis a diferenças estruturais dos sujeitos face à empatia, a escala apresentou uma consistente validade factorial face ao estudo original e ao quadro teórico de referência e uma boa fiabilidade das quatro dimensões que compõem a escala. Está por isso apta a ser aplicada a outras amostras de estudantes e jovens universitários brasileiros e mesmo a outros públicos, continuando o processo de validação da escala e tornando-a mais robusta e credível na avaliação da empatia.
\end{abstract}

Palavras-chave: avaliação da empatia, validade, fiabilidade, estudantes universitários

\begin{abstract}
In this article we present the results of the validation process of the "Test de Empatía Cognitiva y Afectiva (TECA)" for a sample of 256 Brazilian university students. We present the translation and retroversion process of the original scale, the item sensitivity, the factorial validity of the scale and the reliability of its four dimensions and we compared our results with those of the original study. Overall, the items that make up the scale showed to be sensitive to structural differences of the subjects in view of empathy, the scale presented a consistent factorial validity compared to the original study and the theoretical framework of reference and a good reliability of the four dimensions that make up the scale. It is therefore able to be applied to other samples of Brazilian university students and young people and even to other audiences, continuing the process of validating the scale, making it more robust and credible in assessing empathy.
\end{abstract}

Keywords: empathy assessment, validity, reliability, university students

\footnotetext{
${ }^{1}$ Mestre em Educação e Tecnologias Digitais pela Universidade de Lisboa. Professor do Colégio Pedro II, Brasil. Rua Madre Francisca Pia, 640/ casa 2, Petrópolis, RJ, Brasil, CEP: 25.675-222. Tel.: (5524) 99301-0751. E-mail: juliofreit@ gmail.com

${ }^{2}$ Doutorada em Ciências da Educação e Agregada em Educação, especialidade em Tecnologia Educativa pela Universidade de Lisboa. Professora no Instituto de Educação da Universidade de Lisboa. Alameda da Universidade, 1639-013 Lisboa, Portugal. Tel.: +35196245 2549. E-mail: gmiranda@ie.ulisboa.pt

Revista Iberoamericana de Diagnóstico y Evaluación - e Avaliação Psicológica. RIDEP · No58 · Vol.1 · 57-66 · 2021

ISSN: 1135-3848 print /2183-6051online
} 


\section{Introdução}

Entre as competências que serão diferenciais no século XXI e que devem servir de base para o desenvolvimento de uma nova abordagem educacional, uma tem vindo a destacar-se: a empatia. A empatia tem sido considerada em diversas iniciativas, no que Krznaric (2014) assinala como uma "explosão de pensamento e ação empáticos" (p.9). Pechorro et al (2019) defendem que a pesquisa sobre empatia é um tema atual por ter impactos sobre o comportamento social.

Psicólogos e terapeutas consideram a empatia como um elemento essencial para os relacionamentos, sem o qual a própria espécie humana teria tido pouca chance de sobrevivência frente aos desafios enfrentados durante o processo de evolução (VanCleave, 2016). Muitas são as pesquisas sobre o tema e parece certo a importância da empatia para o bem-estar psicológico do ser humano e para o seu comportamento pró-social, o que "favoreceria relações interpessoais à medida que aumentam a probabilidade de gerar uma reciprocidade positiva, solidariedade e um tratamento caloroso". (Balabanian \& Lemos, 2018, p.178)

Segundo Krznaric (2014), um maior grau de empatia tem alta correlação com um maior número de relacionamentos e com relacionamentos de melhor qualidade, e pode "aprofundar as amizades e ajudar a criar outras" (p.20). Essa qualidade vivenciada nos relacionamentos está intimamente ligada à qualidade de vida, à felicidade e, em última instância, a uma vida mais longa (Waldinger \& Schulz, 2010).

Outra questão bastante discutida na literatura sobre o tema é quanto à correlação inversamente proporcional existente entre a empatia e a agressividade e violência (Eisenberg, Eggum, \& Di Giunta, 2010; Feshback \& Feshback, 1969; Mehrabian, 1997). Assim, um nível mais alto de empatia estaria relacionado com um menor grau de agressividade, incluindo o bullying, cyberbullying e mesmo os atos violentos, de cunho sexual ou não, contra as mulheres (Flood \& Pease, 2009; Lisak \& Ivan, 1995).

Talvez por fazer parte da base para relacionamentos saudáveis e não agressivos, a empatia tenha sido constantemente citada por filósofos e cientistas sociais como "uma das maneiras mais eficazes que temos de expandir as fronteiras de nossos universos morais" (Krznaric, 2014, p.21).

Outra área que tem tido um grande foco de estudos sobre a empatia é a clínica, principalmente no que se refere aos transtornos psicológicos, o que resulta, muitas vezes, em uma incapacidade de interagir com outras pessoas, resultado de respostas sociais e emocionais pouco apropriadas. Esses transtornos ocorrem com maior clareza em casos de Síndrome de Asperger. Outros transtornos de personalidade, como o esquizoide, o narcisista, o antissocial e o psicopata também apresentam, como uma de suas características, o baixo nível de empatia dos indivíduos (Fernández-Pinto, López-Pérez, \& Márquez, 2008). Vanaerschot (2004) defende a intervenção empática consistente como um processo de grande importância na construção de significado nesses tipos de transtorno.

Apesar de sua grande relevância, assim como diversos conceitos relacionados à filosofia e à psicologia, não existe um consenso sobre a definição de empatia. Filósofos e pensadores como Leibniz e Rousseau já utilizavam a ideia de colocar-se no lugar do outro, principalmente com o objetivo de serem bons cidadãos (Wispé, 1986, citado por Fernández-Pinto, López-Pérez, \& Márquez, 2008).

Segundo Davis (1996, citado por FernándezPinto, López-Pérez, \& Márquez, 2008), a primeira utilização formal do termo empatia foi feita por Robert Vischer, no século XIX, por meio da palavra alemã "Einfülung", traduzida como "sentir-se dentro de".

Theodor Lipps estende o significado ao termo "Einfülung" incluindo, além da experiência estética dos sentimentos do objeto, vivenciada pelo sujeito, "a percepção do outro, como processo psicológico e como possibilidade ética de atividade de um indivíduo" (Salgado, 2000, p.363).

O processo clássico de definição de empatia levou a uma dicotomia entre uma abordagem afetiva e uma abordagem cognitiva. Embora os primeiros autores tivessem um entendimento afetivo da empatia, não demoraram a surgir aqueles que utilizaram um enfoque mais 
cognitivo. Um dos primeiros a seguir esse caminho foi Köhler, que via a empatia como uma maneira de compreender os sentimentos do outro. (Fernández-Pinto, López-Pérez, \& Márquez, 2008).

A abordagem cognitiva foi dominante entre os autores até o final dos anos 196. Uma comprovação desse fato é oferecida em Hogan (1969). O autor menciona que existia um consenso entre os dicionários da época quanto ao significado de empatia, que seria "a apreensão intelectual ou imaginativa da condição ou estado mental do outro, sem experimentar, realmente, os sentimentos do outro" (p.308). Vê-se, assim, que a perspetiva afetiva não foi somente negligenciada, mas, em alguns casos, também negada.

Um trabalho de profunda importância, nessa década, foi o de Carl Rogers, psicólogo americano que desenvolveu uma metodologia humanista centrada no cliente. Rogers vê a empatia como uma forma de auxiliar os terapeutas a entenderem os pensamentos e significados dos clientes. Percebe-se que a empatia, para Rogers, era formada por processos tanto cognitivos como emocionais, dando uma interpretação mais integrada. Dessa forma, utiliza o termo "compreensão empática" para definir o processo cognitivo, de forma a diferenciá-la da empatia, que pressupõe um evento emocional (Cuff et al, 2014).

Atualmente, seguindo em parte a visão elaborada por Rogers, vemos uma tendência a uma abordagem integradora de empatia, que considera tanto o aspecto afetivo quanto o cognitivo. Davis (1980), em sua busca pelo desenvolvimento de uma ferramenta de medição da empatia, destaca que existe um movimento crescente no sentido de uma integração dessas duas abordagens de pesquisa uma vez que existem indícios de serem partes de um mesmo "sistema interdependente no qual cada parte influencia a outra, e que nunca será totalmente entendido no caso dos esforços serem concentrados em um único aspecto" (p.3).

Como consequência dessa dificuldade em definir empatia é que o seu processo de medição não é simples, como acontece, de forma geral, com as competências socioemocionais. Segundo Sampaio et al (2011), diversos instrumentos e metodologias tem sido utilizados no processo de avaliação da empatia. $\mathrm{O}$ autor divide esses instrumentos em: (a) medidas fisiológicas (condutibilidade e temperatura da pele, frequência cardíaca e respiratória, etc.), (b) índices somáticos (análise de expressões faciais e dos gestos), e (c) entrevistas e escalas de autoavaliação.

Da mesma forma que a evolução do conceito de empatia considerou seus diferentes aspetos (cognitivo, afetivo e situacional), também as escalas de medição de empatia foram desenvolvidas para se adequarem a essas diferentes perspetivas.

Inicialmente, as escalas desenvolvidas por Diamond (1949) e Honga (1969) eram baseadas na visão cognitiva de empatia. Diamond (1949) desenvolveu a primeira escala, a Dymond Rating Test of Insight and Empathy. Nessa escala, os respondentes deviam pontuar determinados adjetivos aplicados a si mesmos e aos outros.

Em um momento posterior, escalas baseadas na visão afetiva tornaram-se bastante utilizadas por pesquisadores. Destaca-se o Questionar Mesure o Emocional Empacha (QMEE), desenvolvido por Mehrabian e Epstein, em 1972. Essa medida, apesar de apresentar redundância entre algumas das suas subescalas, mantém uma boa consistência interna (.84) e, em 1997, foi melhorada por Mehrabian, dando origem à Balanced Emotional Empathy Scale (BEES), com uma consistência interna ligeiramente maior (.87) (Fernández-Pinto, López-Pérez, \& Márquez, 2008).

Tendo uma perspetiva mais integradora de empatia, pode-se destacar o Índice de Reatividade Interpessoal (IRI), desenvolvido por Davis (1980), o Quociente de Empatia (QE), desenvolvido por Baron-Cohen e Weelbright (2004), possuindo consistência interna adequada (.85) e uma terceira escala, desenvolvida por López-Pérez, FernándezPinto y Abad (2008), para a população de língua hispânica, designada por Teste de Empatia Cognitiva e Afetiva (TECA).

A TECA tem consistência interna de .86, contemplando, em suas subescalas, a Adoção de Perspetiva, a Compreensão Emocional, ambas representando a Empatia Cognitiva (EC), o Estresse Empático e, como diferencial frente às demais escalas, inclui a Alegria Empática, ambas representando a Empatia Emocional (EE), que é a capacidade de compartilhar as emoções positivas de outra pessoa. 
Apesar de ter sido traduzida para o português de Portugal por Palhoco e Afonso (2011), "com base nas Diretrizes de Adaptação de Testes Psicológicos (International Test Commission [ITC], 2010)" (p.140), a escala não havia sido traduzida nem validada para o português do Brasil, o que se fazia necessário pelas características linguísticas, sociais e culturais diferenciadas das duas nações.

Assim, o objetivo do presente trabalho, como parte integrante de uma dissertação de mestrado sobre a possibilidade de desenvolvimento da empatia por meio de um curso ministrado em regime de e-learning, foi realizar a tradução e adaptação para o português do Brasil, bem como sua validação para utilização em meio universitário.

\section{Método}

\section{Participantes}

A fim de validar a escala, utilizou-se uma amostra de conveniência de jovens e adultos, alunos da Instituição de Ensino Superior (IES) na qual foi realizada, posteriormente, a pesquisa de campo para a dissertação de mestrado. Responderam à escala TECA 303 alunos. Entretanto, foram retirados da amostra sujeitos que não preencheram todos os campos da escala, o que resultou em uma amostra final de 256 alunos. A amostra era composta por 182 mulheres $(71,1 \%)$ e 74 homens $(28,9 \%)$. A idade média dos respondentes era de 21,58 anos (desvio padrão de 4,88), com idade mínima de 17 anos e máxima de 54.

Quanto à divisão dos respondentes por curso frequentado na IES, 84 eram alunos de Administração, 26 de Enfermagem, 49 de Medicina, 31 de Odontologia, 54 de Psicologia e 12 de Recursos Humanos.

\section{Medidas}

O TECA contém, originalmente, 33 frases afirmativas que devem ser classificadas segundo a escala de Likert de 5 pontos, marcando 1 para frases afirmativas com as quais o respondente discorde totalmente e 5 para frases afirmativas com as quais o respondente concorde totalmente.

O TECA possui quatro subescalas: adoção de perspetiva ( 8 frases afirmativas), compreensão emocional (9 frases afirmativas), estresse empático ( 8 frases afirmativas), e alegria empática (8 frases afirmativas), sendo sua pontuação máxima de 165 pontos (33 frases afirmativas x 5 pontos). (López-Pérez, Fernández-Pinto, \& Abad, 2008)

A Adoção de Perspetiva (AP) "faz referência à capacidade intelectual ou imaginativa de colocar-se no lugar de outra pessoa" (p.8). A Compreensão Emocional (CE) "refere-se à capacidade de reconhecer e compreender os estados emocionais, as intenções e as impressões dos outros" (p.9). O Estresse Empático (EE) "é a capacidade de compartilhar as emoções negativas de outras pessoas, de se sintonizar emocionalmente" (p.9). A Alegria Empática (AE) "refere-se à capacidade de compartilhar as emoções positivas de outras pessoas" (p.10).

\section{Procedimentos}

Por respeito aos princípios éticos associados à investigação, tanto os autores do estudo original quanto a editora detentora dos diretos de publicação da TECA foram contactados e autorizaram a publicação da adaptação da escala original para o português do Brasil.

No processo de tradução de um questionário é desejável que se mantenha o mais possível o sentido original dos seus diferentes itens, mas também as suas instruções de preenchimento e escalas de avaliação (Moreira, 2004). Dessa forma, inicialmente foi feita uma tradução livre da escala pelos autores da pesquisa, que foi revista por especialistas na área temática, tendo como objetivo ajudar na clarificação dos itens, a fim de evitar mal-entendidos por parte dos sujeitos. Obteve-se também o auxílio de um professor doutor em Pedagogia da Aprendizagem Humana, falante nativo da língua portuguesa, atuando desde 2006 com a língua espanhola, no México, oferecendo uma contribuição significativa para a coerência e a especificação conceptual dos termos, permitindo uma melhor compreensão dos termos semelhantes entre o espanhol e o português do Brasil, cujos sentidos são distintos nas duas línguas ("falsos cognatos").

Todos os participantes colaboraram voluntariamente, tendo sido informados sobre os objetivos meramente acadêmicos da pesquisa, bem como sobre as questões éticas relativas à 
confidencialidade e anonimato dos dados pessoais. Os dados sofreram tratamento estatístico, e nenhum dado individual foi publicado.

A aplicação da escala decorreu em contexto de grupo presencial, em sala de aula. O aplicador treinado explicava a todos os presentes o propósito da pesquisa mantendo-se à disposição durante toda a aplicação. O tempo médio de aplicação foi de 20 minutos em cada grupo. Para o tratamento dos dados foi utilizado o programa estatístico IBM SPSS Statistics, versão 23. Para se garantir a credibilidade do instrumento de medida, foram analisadas a sensibilidade dos itens utilizando a assimetria e a curtose, a validade fatorial e a fiabilidade obtida pelo Alfa de Cronbach.

\section{Resultados}

\section{Análise dos Dados}

Primeiramente, foram analisadas as estatísticas descritivas dos dados, por subescala e dos dados consolidados (Quadro 1).

Quadro 1. Estatística descritiva dos resultados obtidos junto dos sujeitos da amostra

\begin{tabular}{|c|c|c|c|c|c|}
\hline & AP & $\mathrm{CE}$ & $\mathrm{EE}$ & $\mathrm{AE}$ & TOTAL \\
\hline $\mathrm{N}$ & 256 & 256 & 256 & 256 & 256 \\
\hline Média & 29.87 & 31.64 & 26.81 & 35.28 & 123.60 \\
\hline $\begin{array}{l}\text { Desvio } \\
\text { Padrão }\end{array}$ & 4.93 & 5.26 & 6.40 & 3.98 & 14.36 \\
\hline Assimetria & -.48 & .03 & -.10 & -1.04 & -.337 \\
\hline Curtose & .00 & -.32 & -.57 & 1.01 & -.223 \\
\hline Mínimo & 14 & 18 & 10 & 21 & 85 \\
\hline Máximo & 40 & 45 & 40 & 40 & 157 \\
\hline
\end{tabular}

Em seguida, realizou-se um teste de sensibilidade de todos os itens da escala. Para isso, foram utilizadas as medidas de assimetria e curtose, cujos resultados são apresentados no Quadro 2.

Dos 33 itens testados, 20 itens possuem elevada sensibilidade, tendo seus valores próximos a zero. Segundo Marôco (2007), somente valores com Assimetria acima de 3 e Curtose acima de 7 apresentam problemas graves de sensibilidade. Dos restantes 13 itens, somente o item 4 possui curtose maior do que 7 , porém com Assimetria menor do que 3. Os demais itens apresentaram valores acima de 1, indicando menor capacidade discriminativa, porém não tendo problemas graves de sensibilidade.
Quadro 2. Resultado do Teste de Sensibilidade -

\begin{tabular}{|c|c|c|}
\hline \multicolumn{3}{|c|}{ Assimetria e Curtose } \\
\hline \multicolumn{3}{|c|}{ Teste de Sensibilidade } \\
\hline & SK & $\mathrm{Ku}$ \\
\hline Item 1 & -.248 & -.067 \\
\hline Item 2 & -2.061 & 4.547 \\
\hline Item 3 & -.504 & -.482 \\
\hline Item 4 & -2.694 & 7.846 \\
\hline Item 5 & -.077 & -1.303 \\
\hline Item 6 & -.818 & -.073 \\
\hline Item 7 & -.3 & -.615 \\
\hline Item 8 & -.843 & -.454 \\
\hline Item 9 & -1.955 & 3.673 \\
\hline Item 10 & .004 & -1.165 \\
\hline Item 11 & -.251 & -.585 \\
\hline Item 12 & -.255 & -1.298 \\
\hline Item 13 & -1.374 & 1.427 \\
\hline Item 14 & -.315 & -.831 \\
\hline Item 15 & -.794 & -.114 \\
\hline Item 16 & -1.974 & 3.988 \\
\hline Item 17 & -.519 & -.561 \\
\hline Item 18 & 463 & -1.016 \\
\hline Item 19 & -.867 & .111 \\
\hline Item 20 & -1.175 & .996 \\
\hline Item 21 & -1.336 & .638 \\
\hline Item 22 & -.869 & .175 \\
\hline Item 23 & .085 & -1.254 \\
\hline Item 24 & .074 & -.936 \\
\hline Item 25 & -1.986 & 3.286 \\
\hline Item 26 & -.606 & -.462 \\
\hline Item 27 & -.265 & -.467 \\
\hline Item 28 & -.364 & -.804 \\
\hline Item 29 & -.496 & -.727 \\
\hline Item 30 & -1.238 & .452 \\
\hline Item 31 & -.736 & .144 \\
\hline Item 32 & -.132 & -.916 \\
\hline Item 33 & -.464 & -.565 \\
\hline
\end{tabular}

Nota. Fonte: SPSS

\section{Validade Fatorial}

Primeiramente, foi realizada a análise de fatorabilidade dos dados obtidos, que foi confirmada com $\mathrm{KMO}=.794$ (bom) e o teste de esfericidade de Bartlett com $p<.01$, indicando que a matriz de dados é favorável à análise fatorial, conforme apresentado no Quadro 3.

A fim de validar a escala, verificando se a amostra se comportava da mesma forma que a validação original, foi realizado uma análise 
Quadro 3. Resultado este KMO e de esfericidade de Bartlett realizado no SPSS

\begin{tabular}{lcc}
\hline \multicolumn{2}{c}{ Teste de KMO e Bartlett } \\
\hline Medida Kaiser-Meyer-Olkin de adequação de amostragem & .794 \\
\hline \multirow{2}{*}{ Teste de esfericidade de Bartlett } & Aprox. Qui-quadrado & 1919.576 \\
& gl & 528 \\
& Sig. & .000 \\
\hline
\end{tabular}

Nota. Fonte: SPSS

Quadro 4. Matriz de Estruturas dos Itens da Escala TECA, EFCP com Rotação Oblíqua

\begin{tabular}{|c|c|c|c|c|}
\hline \multirow[b]{2}{*}{ Item } & \multicolumn{4}{|c|}{ Componente } \\
\hline & $\begin{array}{c}\text { Adoção de Perspetiva } \\
\text { (AP) }\end{array}$ & $\begin{array}{l}\text { Compreensão Emocional } \\
\text { (CE) }\end{array}$ & $\begin{array}{c}\text { Estresse Empático } \\
\text { (EE) }\end{array}$ & $\begin{array}{c}\text { Alegria Empática } \\
\text { (AE) }\end{array}$ \\
\hline 2 & & & & .476 \\
\hline 4 & & & & .619 \\
\hline 8 & & & & .429 \\
\hline 16 & & & & .668 \\
\hline 19 & & & & .629 \\
\hline 21 & & & & .657 \\
\hline 25 & & & & .420 \\
\hline 3 & & & .394 & \\
\hline 5 & & & .677 & \\
\hline 12 & & & .620 & \\
\hline 18 & & & .647 & \\
\hline 22 & & & .427 & \\
\hline 23 & & & .760 & \\
\hline 28 & & & .616 & \\
\hline 30 & & & .604 & \\
\hline 1 & & .509 & & \\
\hline 7 & & .568 & & \\
\hline 10 & & .374 & & \\
\hline 13 & & .490 & & \\
\hline 14 & & .485 & & \\
\hline 24 & & .487 & & \\
\hline 27 & & .657 & & \\
\hline 31 & & .477 & & \\
\hline 33 & & .617 & & \\
\hline 6 & .565 & & & \\
\hline 9 & .362 & & & \\
\hline 11 & .502 & & & \\
\hline 15 & .669 & & & \\
\hline 17 & .372 & & & \\
\hline 20 & .500 & & & \\
\hline 26 & .594 & & & \\
\hline 29 & .558 & & & \\
\hline 32 & .509 & & & \\
\hline \multicolumn{5}{|c|}{ Variância Explicada } \\
\hline $\mathrm{AE}$ & $16.899 \%$ & & & \\
\hline $\mathrm{EE}$ & $7.974 \%$ & & & \\
\hline $\mathrm{CE}$ & $5.583 \%$ & & & \\
\hline AP & $5.208 \%$ & & & \\
\hline Total & $35.664 \%$ & & & \\
\hline
\end{tabular}

fatorial de componentes principais (EFCP) com rotação oblíqua (oblimin) com normalização de Kaiser, mesmo método utilizado na validação original. Este procedimento teve como finalidade verificar quais os itens que pertenciam a cada subescala e se a divisão encontrada na amostra coincidia com a divisão da validação original. Apesar da validação original da TECA ter utilizado o valor de .30 como carga fatorial mínima, segundo Fávero et al (2009), para uma amostra com 250 casos, o indicado é uma carga fatorial mínima de .35. Dessa forma, assumimos essa carga fatorial mínima no presente trabalho, eliminando todos os demais itens abaixo desse valor. 
O Quadro 4 apresenta a matriz de estruturas resultante do teste, onde se destacam as saturações maiores do que .35 dos itens divididos nas quatro subescalas.

Comparando os resultados obtidos na validação original com os resultados da presente pesquisa, foram identificadas discrepâncias em três itens $(8,9$ e 22$)$.

$\mathrm{O}$ item 8 pertence, originalmente, à subescala Estresse Empático, porém em nossa amostra o item foi classificado como pertencente à subescala Alegria Empática. Esse fato pode decorrer de uma interpretação errônea por parte dos respondentes, oriunda de uma tradução e/ou adequação linguística inadequada. Somado a isso, trata-se de um item invertido, fato que alguns autores têm mencionado problemas, "pois estes exigem mais atenção e compreensão por parte do participante" (Kobarg, Vieira, \& Vieira, 2010, p.81). Uma vez que não percebemos uma relação conceitual entre o item e a subescala Alegria Empática, retirou-se o item da escala final.

O item 9, na validação original, pertencia à subescala Alegria Empática. Em nossa amostra, o item relacionou-se com a subescala Adoção de Perspetiva. Nesse caso, entendemos haver uma relação conceitual com as duas subescalas. Se, por um lado, o sentimento de outra pessoa pode despertar um sentimento positivo, caracterizando a alegria empática, por outro, esse sentimento positivo pode derivar do exercício cognitivo de imaginar-se naquela situação e do desejo de ser bem recebido. Dessa forma, escolhemos por manter esse item na subescala Adoção de Perspetiva.

O último caso de discrepância entre a validação original e a realizada nessa pesquisa refere-se ao item 22. Nesse caso específico, o item mostrou saturação para a subescala Estresse Empático (.427). Porém, não existe justificativa conceitual para a saturação desse item na subescala Estresse Empático, uma vez que é simulada uma situação onde ocorrem emoções positivas e o estresse empático demanda o compartilhamento de emoções negativas. Dessa forma, optamos por retirar esse item da escala adaptada.

A escala TECA-BR adaptada ficou com 31 itens, sendo divididas as subescalas da seguinte forma: Adoção de Perspetiva (9 itens);
Compreensão Emocional (9 itens); Estresse Empático ( 7 itens); e Alegria Empática (6 itens). A nova pontuação máxima é de 155 (31 x 5).

\section{Fiabilidade}

A medida utilizada para verificar a confiabilidade da escala TECA-BR, traduzida e adaptada, foi o alfa de Cronbach, medida também utilizada por López-Pérez, Fernández-Pinto y Abad no teste de validade original da escala. $\mathrm{O}$ Quadro 5 apresenta os resultados obtidos quanto à consistência interna em cada subescala do TECA.

Quadro 5. Consistência Interna das Subescalas do TECA

\begin{tabular}{lcc}
\hline Subescalas & No de Itens & Alfa de Cronbach \\
\hline AP & 9 & .71 \\
CE & 9 & .67 \\
EE & 7 & .76 \\
AE & 6 & .68 \\
TOTAL & 31 & .82 \\
\hline Nota. Fonte: SPSS & &
\end{tabular}

Percebe-se que as subescalas, exceto CE e AE, possuem valores iguais ou superiores a .7, considerado satisfatório para utilização, e a escala como um todo possui coeficiente .82 , considerado como valores recomendados (Cunha, Almeida Neto, \& Stackfleth, 2016). O valor de .67 da subescala $\mathrm{CE}$ e .68 da subescala $\mathrm{AE}$ são pouco inferiores ao limite mínimo e podem ser explicados pelo baixo número de itens das subescalas e pela utilização de uma amostra homogênea de testados, uma vez que todos os casos são alunos da mesma instituição de ensino superior, com idades e nível sociocultural próximos (Cunha, Almeida Neto \& Stackfleth, 2016).

Dessa forma, as subescalas e a escala como um todo mostram uma consistência interna aceitável, ainda mais quando se considera o número reduzido de itens em sua composição.

\section{Validade Convergente e Discriminante}

Segundo Ghadi et al (2012) e Valentini \& Damásio (2016), a validade convergente pode ser compreendida como a convergência entre os itens que compõem um fator, havendo um relacionamento significativo entre eles. A validade convergente pode ser medida pela Confiabilidade Composta(CC). Ainda segundo os autores, valores de $\mathrm{CC}$ iguais ou superiores a .7 indicam a validade convergente para o fator. 
Quadro 6. Confiabilidade Composta (CC) das Subescalas do TECA

\begin{tabular}{lcc}
\hline Subescalas & No de Itens & $\begin{array}{c}\text { Confiabilidade } \\
\text { Composta }(\mathrm{CC})\end{array}$ \\
\hline $\mathrm{AP}$ & 9 & .77 \\
$\mathrm{CE}$ & 9 & .77 \\
$\mathrm{EE}$ & 7 & .81 \\
$\mathrm{AE}$ & 6 & .77 \\
\hline Nota. Fonte: Próprio Autor
\end{tabular}

O Quadro 6 apresenta os resultados de CC obtidos para cada fator.

A validade discriminante pode ser vista como o complemento da validade convergente, uma vez que mede o quanto os itens de um determinado fator não estão correlacionados a outros fatores. Para avaliar a validade discriminante verificamos se a Variância Extraída Média de cada fator era maior do que o quadrado das correlações entre esses fatores $\left(\phi^{2}\right)$.

O Quadro 7 apresenta os resultados dessa comparação para todos os fatores, que atestam essa condição. Todas as comparações resultaram em $\Delta\left(\right.$ VEM- $\left.\phi^{2}\right) \geq$. Dessa forma, podemos assumir que as condições para a validade discriminante são atendidas para a ferramenta de medição adaptada.

\begin{tabular}{lcc}
\multicolumn{4}{c}{ Quadro 7. Diferença entre a Variância Extraída } \\
Média (VEM) de cada fator e o quadrado das \\
\multicolumn{4}{c}{ correlações entre esses fatores $\left(\phi^{2}\right)$} \\
\hline \multicolumn{2}{c}{ Correlação entre: } & \multicolumn{2}{c}{ Subescalas } & $\Delta\left(\right.$ VEM $\left.-\phi^{2}\right)$ \\
\hline \multirow{2}{*}{ AP-CE } & AP & .22 \\
& $\mathrm{CE}$ & .22 \\
AP-EE & $\mathrm{AP}$ & .26 \\
& $\mathrm{EE}$ & .38 \\
AP-AE & $\mathrm{AP}$ & .24 \\
& $\mathrm{AE}$ & .31 \\
CE-EE & $\mathrm{CE}$ & .25 \\
& $\mathrm{EE}$ & .37 \\
CE-AE & $\mathrm{CE}$ & .22 \\
& $\mathrm{AE}$ & .29 \\
EE-AE & $\mathrm{EE}$ & .34 \\
\hline
\end{tabular}

Nota. Fonte: Próprio Autor

\section{Discussão}

O principal objetivo do presente estudo, como parte maior de uma dissertação de conclusão de mestrado, foi validar e adaptar o Test de Empatía Cognitiva e Afetiva (TECA) para utilização entre estudantes universitários brasileiros, por meio da análise estatística dos resultados obtidos pela aplicação do TECA em uma amostra de estudantes brasileiros.
Os itens da TECA apresentam, de forma geral, boa sensibilidade, com exceção do item 4, com valor de curtose elevado. Apesar disso, o item apresenta peso fatorial >.6, sendo estatisticamente significativo, e a escala possui um alfa de Cronbach bom $(\alpha=.82)$, optou-se por manter este item na escala final.

Após a realização de análise fatorial de componentes principais com rotação oblíqua (oblimin) na amostra, houve discrepâncias na classificação dos itens 8,9 e 22 frente aos resultados obtidos na validação original. Assim, optou-se pela exclusão dos itens 8 e 22 por lacuna de sustentação teórica que demonstrasse relação entre os itens e as subescalas nas quais foram classificados, respectivamente, como AE e EE. Houve também a reclassificação do item 9 de $\mathrm{AE}$ para AP por entendermos haver uma relação conceitual com as duas subescalas

Dessa forma, a escala TECA-BR adaptada ficou com 31 itens, da seguinte forma: AP - 9 itens; CE - 9 itens; EE - 7 itens; e AE - 6 itens, sendo a nova pontuação máxima de 155 (31 x 5).

Finalizando para a validação da escala, foi realizado um teste de fiabilidade utilizando o alfa de Cronbach. A escala total apresentou coeficiente igual a .82, sendo considerado satisfatório para utilização (igual ou superior a .7), assim como as subescalas AP (.71) e EE (.76). As subescalas CE e AE apresentaram valores pouco inferiores a esse limite (.67 e .68 , respetivamente) que podem ser explicados pelas características da amostra, não comprometendo a validade do instrumento. Foram realizados também testes nos quais ficaram garantidas a Validade Convergente e a Validade Discriminante.

Conforme referido no presente trabalho, a medição de competências socioemocionais apresenta expressivos desafios e, poder contar com uma ferramenta traduzida e adaptada para o português brasileiro, tem considerável relevância, principalmente considerando o público de jovens e adultos, no nosso caso, estudantes universitários, focos do TECA.

$\mathrm{O}$ processo de validação de um instrumento de medida não se encerra e deve ser ampliado por meio de outras amostras, a fim de identificar se os resultados encontrados são coerentes, o que propicia maior credibilidade ao instrumento. 


\section{Referências}

Balabanian, C., \& Lemos, V. N. (2018). Desarrollo y estudio psicométrico de una escala para evaluar conducta prosocial en adolescentes. Revista Iberoamericana de Diagnóstico y Evaluación - e Avaliação Psicológica, $\quad 48(3), \quad$ 177-188. doi:1.21865/RIDEP48.3.15

Baron-Cohen, S., \& Wheelwright, S. (2004). The Empathy Quotient: An investigation of adults with asperger syndrome or high functioning autism, and normal sex differences. Journal of Autism and Developmental Disorders 34, 163175.

doi:1.1023/B:JADD.0000022607.19833.00

Cuff, B., Brown, S. J., Taylor, L., \& Howat, D. (2014). Empathy: A review of the concept. Emotion Review. Retirado de http://emr.sagepub.com/content/early/2014/12 /01/1754073914558466. doi:1.1177/1754073914558466

Cunha, C. M., de Almeida Neto, O. P., \& Stackfleth, R. (2016). Principais métodos de avaliação psicométrica da validade de instrumentos de medida. Revista de Atenção à Saúde, 14(47), 75-83.

doi:1.13037/ras.vol14n47.3391

Davis, M. H. (1980). A multidimensional approach to individual differences in empathy. Journal of Personality and Social Psychology, 10(85), 1-19.

Dymond, R. F. (1949). A scale for the measurement of empathic ability. Journal of Consulting Psychology, 13(2), 127-133. doi:1.1037/h0061728

Eisenberg, N., Eggum, N. D., \& Di Giunta, L. (2010). Empathy-related responding: Associations with prosocial behavior, aggression, and intergroup relations. Social Issues and Policy Review, 4(1), 143-18. doi:1.1111/j.1751-2409.201.0102.x

Fávero, L. P. L., Belfiore, P. P., Silva, F. L. da, \& Chan, B. L. (2009). Análise de dados: modelagem multivariada para tomada de decisões. Rio de Janeiro: Elsevier.

Fernández-Pinto, I., López-Pérez, B., \& Márquez, M. (2008). Empatía: Medidas, teorías y aplicaciones en revisión. Anales de Psicología, 24(2), 284-298. doi:1.6018/analesps
Feshbach, N. D., \& Feshbach, S. (1969). The relationship between empathy and aggression in two age groups. Developmental Psychology, 1(2), 102-107. doi:1.1037/h0027016

Flood, M., \& Pease, B. (2009). Factors influencing attitudes to violence against women. Trauma, Violence, \& Abuse, 10(2), 125-142. doi:1.1177/1524838009334131

Ghadi, I., Alwi, N. H., Bakar, K. A., \& Talib, O. (2012). Construct validity examination of critical thinking dispositions for undergraduate students in University Putra Malaysia. Higher Education Studies, 2(2), 138-145. ISSN: ISSN-1925-4741

Hogan, R. (1969). Development of an empathy scale. Journal of Consulting and Clinical Psychology, 33(3), 307. doi:1.1037/h0027580

Lisak, D., \& Ivan, C. (1995). Deficits in intimacy and empathy in sexually aggressive men. Journal of Interpersonal Violence, 10(3), 296308. doi:1.1177/088626095010003004

Kobarg, A. P. R., Vieira, V., \& Vieira, M. L. (2010). Validação da escala de lembranças sobre práticas parentais (EMBU). Avaliação Psicologica: Interamerican Journal of Psychological Assessment, 9(1), 77-85. ISSN:1677-0471

Konrath, S., \& Grynberg, D. (2013). The positive (and negative) psychology of empathy. The neurobiology and psychology of empathy. Hauppauge, NY: Nova Biomedical Books.

Krznaric, R. (2014). O Poder da empatia: A arte de se colocar no lugar do outro para transformar o mundo. Rio de Janeiro: Zahar.

Marôco, J. (2007). Análise estatística com utilização do SPSS. Lisboa: Edições Sílabo.

Mehrabian, A. (1997). Relations among personality scales of aggression, violence, and empathy: Validational evidence bearing on the Risk of Eruptive Violence Scale. Aggressive Behavior, 23(6), 433-445. doi:1.1002/(SICI)10982337(1997)23:6<433::AID-AB3>3..CO;2-H

Moreira, V. (2004). O método fenomenológico de Merleau-Ponty como ferramenta crítica na pesquisa em psicopatologia. Psicologia: Reflexão e Crítica, 17(3), 447-456. doi:1.1590/S0102-79722004000300016

Palhoco, A. R., \& Afonso, M. J. (2011). A empatia e a percepção de emoções em 
estudantes de psicologia e psicoterapeutas. Estudos Interdisciplinares em Psicologia, 2(2), 133-153. doi:1.5433/2236-6407.2011v2n2p133

Pechorro, P., Jesus, S. N., Kahn, R. E., Gonçalves, R. A., \& Barroso, R. (2018). A Versão Breve da Escala de Empatia Básica numa amostra escolar de jovens portugueses: Validade, fiabilidade e invariância. Revista Iberoamericana de Diagnóstico y Evaluación - e Avaliação, 49(4), 157-169. doi:1.21865/RIDEP49.4.13

Salgado, M. (2000). Del valor estético de la empatía al negocio inteligente de las emociones: La psicología estética de Theodor Lipps a las puertas del tercer milenio. Revista de Historia de la Psicología, 21(2-3), 359372. ISSN 0211-0040

Sampaio, L. R., Guimarães, P. R. B., dos Santos, C. P., Formiga, N. S., \& Menezes, I. G. (2011). Estudos sobre a dimensinalidade da empatia: Tradução e adaptação do Interpersonal Reactivity Index (IRI). Psico, 42(1), 67-76. e-ISSN: 1980-8623

Valentini, F., \& Damásio, B. F. (2016). Variância média extraída e confiabilidade composta: Indicadores de precisão. Psicologia: Teoria e Pesquisa, 32(2). doi:1.1590/0102-3772e322225

Vanaerschot, G. (2004). It takes two to tango: On empathy with fragile processes. Psychotherapy: Theory, Research, Practice, Training, 41(2), 112-124. doi:1.1037/0033-3204.41.2.112

VanCleave, D. S. (2016). Contributions of neuroscience to a new empathy epistemology: Implications for developmental training. Advances in Social Work, 17(2), 369-389. doi:1.18060/21087

Waldinger, R. J., \& Schulz, M. S. (2010). What's love got to do with it?: Social connections, perceived health stressors, and daily mood in married octogenarians. Psychology and Aging, 25(2), 422. 